\title{
Students' Vocational Skills Training of Road and Bridge Engineering
}

\author{
XIA Xiaohui and YANG Yixi \\ Hunan communication polytechnic, Hunan Changsha 410132 \\ hnxiaxiaohui@yeah.net
}

Keywords: vocational skills, road and bridge engineering, technology education

\begin{abstract}
The purpose of this study is to explore the engineering problem finding ability of high school students of road and bridge engineering. Students were categorized and evaluated qualitatively based on school and gender of respondent. Results indicate that the most consistent predictor of creativity in engineering problem finding scenarios was the number of advanced science classes. The qualitative results showed striking differences in the responses from students. Results show educators need to become more aware of the importance of problem finding in engineering and better encourage the development of problem finding skills among their students.
\end{abstract}

\section{Introduction}

Technology education in China like industrial arts and manual training before it, has traditionally focused on domain knowledge and production skills rather than on intellectual processes. Among other indicators that the American system of public education was on a dangerous path it was reported that high school students were lacking in intellectual skills, such as problem solving, which should be expected of them. Although technology educators in China have been slow to fully integrate a design process in their curriculum other parts of the world have truly embraced design as a foundation for technology education. The advent and subsequent revisions of the national curriculum in China have firmly entrenched the idea of design and problem solving within their technology curriculum, though some progress is being made. In recent years the field of technology education in China has been moving towards a more engineering and design focused curriculum. As road and bridge engineering curriculum continue to propagate through schools there will need to be continued research which aids us in understanding student problem solving both in how problem solving can be nurtured in students as well as appropriate methods for the assessment of problem solving. The study presented here focuses on describing the state of engineering problem finding at three high schools.

\section{Problems on Vocational Skills Training of Road and Bridge Engineering}

One of the major issues teachers have with problem based learning and the type of team oriented design problems that are encouraged in engineering curriculum is the difficulty in assessing student performance. Design is so often a subjective assessment, something that can be difficult to justify to parents and students even when rubrics are used. Furthermore, the group process which is frequently used in these scenarios can leave a teacher with little information about the thinking which went into a design and which might be of use in determining a grade. The use of problem posing as an alternative evaluation method has the potential to give teachers some additional information about student thinking and the creative process which led them to a specific design, something which would be of certain interest for instructors who are frequently asking students how they arrived at a particular design.

One of the challenges in studying creativity is that of predicting and measuring creative performance, especially on a large scale. Much of the older research on predicting creativity used standard measures of divergent thinking such as the Uses. Although variables such as personality, values, intrinsic motivation and discovery orientation have been suggested as predictors of creativity these, by themselves, leave much variance to be accounted for. Difficulty in studying creativity comes from the difficulty of defining it. In other words, all measures of creativity come down to a 
judgment call at some point. There is an individual or panel of individuals who must determine if something is creative or not. Even under the best circumstances these evaluators are bound by the current values and norms as they determine creativity. It is even true that in some cases individuals who were not considered particularly creative by themselves of their contemporaries are later judged to be creative based on their contribution to overall development.

While it is the case that many engineers are initially drawn to the profession by an interest in gaining the ability to solve real-world problems, problems which would require creative solutions, these are not the types of problems which they frequently encounter in the engineering schools. In contrast, there is a concern among some engineering educators that the engineering science based curriculum which pervades most schools of engineering has the opposite effect, that of stifling creativity. Noting that engineers must frequently work within the confines of legal requirements, cost requirements and material properties. There remains a need to foster creativity in these students. One way they propose doing this is though the integration of capstone projects. It is their belief that these projects simulate realistic design scenarios and improve students' understanding, motivation and creativity.

There is also a concern among the engineering profession that problem solving has become too analytical and procedural. The concern is that students in engineering schools are learning "engineering by formula" and are being taught with neat problems which will never be seen in the real world and which do not transfer well to the complex nature of real problems. This "plug-and-chug" method of engineering is not only what industries hiring engineers want to avoid; it is also a less efficient method of arriving at a solution. Students are familiarized with working in real-world contextualized situations throughout their engineering school careers and that creativity in design is encouraged rather than discouraged. This is not to say that engineering schools are all discouraging creativity or the creative design process. Quite the contrary, some engineering schools have made tremendous efforts to include creativity within their curriculum. This type of problem finding, though not explicitly included in the course, would seem to be encouraged as a creative solution to the problem. There is one project in the course where the students are expected to find and solve their own engineering problem. Towards the end of the course students are asked to form groups and then select and solve an electrical engineering problem which has not yet been solved or to make a major improvement in an existing solution.

\section{Methods of Skills Training of Road and Bridge Engineering Students}

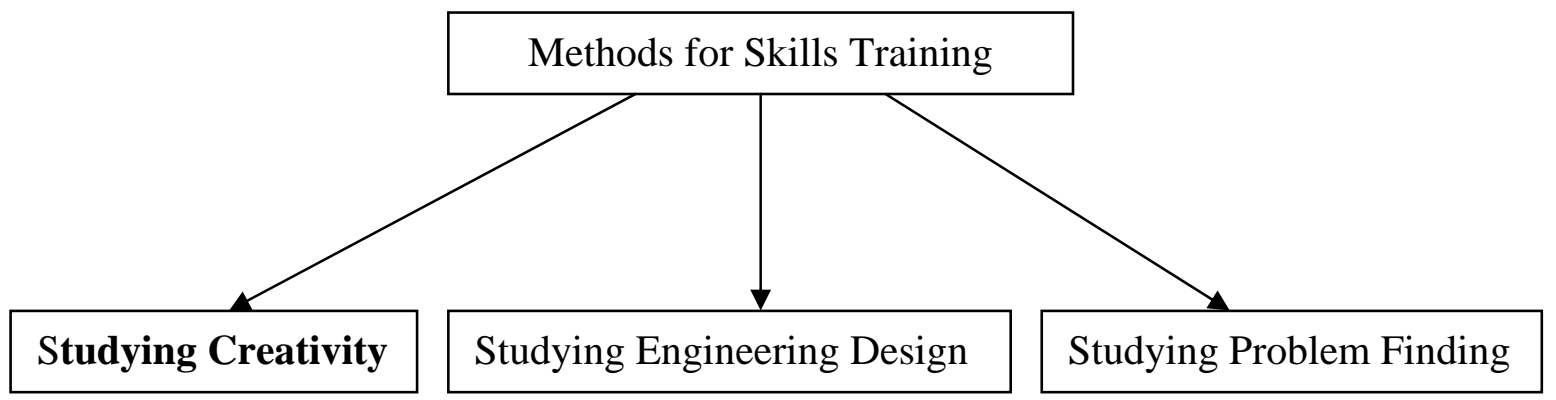

Figure. 1 methods of skills training of road and bridge engineering students

Studying Creativity. Interest in the measurement of creativity is something which developed primarily in the second half of the twentieth century. Prior to this creativity had been considered the domain of the genius and a mysterious process which could not be fully understood. If creativity was an inherited trait or one received by chance there would be little purpose in measuring it beyond the worth of the products created. Once creativity began to be viewed as a valuable social trait to which all might aspire and interest arose in the teaching and nurturing of creativity there was an explosion of interest in the measurement of creativity so that these efforts to teach and nurture creativity might be evaluated. Before one can begin to measure creativity there must be a definition which clarifies what 
about creativity is to be measured. If original, inventive and novel ideas are the outcomes of creativity we are still left at somewhat of a loss on how to measure these ideas as they are, at least to some extent, subjective measures which vary not only by evaluator but by time and place.

Studying Engineering Design. Although many researchers have studied the engineering design process, much of their research has been confined to structured interviewing, observation and verbal protocol analysis. While these types of studies can provide a great depth of knowledge about the design process they tend to focus on understanding the process through a very small group of designers and not on surveying the abilities of a large group. The goal of this type of research seems more to be the identification and exploration of a design process or model than determining that is good at design or why they are. Furthermore, although there is recognition among design scholars that engineering design is a type of problem solving there have been few studies which specifically examine this link or discuss the problem finding aspect of engineering design. The most common method for studying the design process appears to be thinking aloud verbal protocol analysis. This type of research involves participants verbalizing thoughts and actions as they work through a design problem. Instead of asking the participant to reflect on the design process and thus distract them and introduce their own thoughts the think aloud process is designed to provide as little interruption as possible and allow conclusions to be drawn about the cognitive processes being employed by the participant as they go about their task. The recorded protocols are transcribed then segmented and coded by the researcher in an effort to analyze the design process or methodology.

Studying Problem Finding. Research on problem finding has not been solely the domain of either quantitative or qualitative research though there is some preference for quantitative methods. The preference is understandable when it is considered that research on problem finding grew out of research on problem solving undertaken by educational psychologists. Even those studies which employ data collection methods often associated with qualitative studies such as interviews and observation are frequently quantified for analysis of problem finding. Much of the foundational research in problem finding has been conducted using what might be called quantified observation. This technique relies on observation of the problem finding process in a way which is eventually quantified for further analysis.

\section{Discussion}

The increasing importance of engineering and engineering design within the technology education field presents new challenges for the technology educator, particularly as it related to the development and assessment of new curriculum. Successful engineers are able to deal with ambiguity, uncertainty and understand the broad implications of their designs and successful engineering education curriculum addresses these areas. Engineering design has been presented as a specific case of problem solving which begins with the problem finding or scoping process and which drives the selection and quality of the ultimate solution. It is suggested that classroom activities such as reflection, writing, visualization, brainstorming and non-argumentative conversation support the development of creative problem solving skills and creative thinking. In much the same way that creativity has formed a backdrop for the study of engineering and design in the professional fields so too must creativity inform the field of engineering and technology education. Students seem to be both motivated and interested in the ability of engineering and design to solve real-world problems in creative ways. Although there has been a focus on engineering science in schools of engineering there is some evidence of backlash against this and technology education must be careful about embracing the fact-based and exact nature of science and engineering when the ill-structured and complex problems which we expect and hope that engineers are able to solve are frequently aided by the inclusion of creativity rather than mere technical skill.

\section{References}

[1] H. Roman: The Technology Teacher, Vol. 60 (2001), p. 31-33. 
[2] M. Shodell: The American Biology Teacher, Vol. 57 (1995), p. 278-281.

[3] E. Silver and J. Cai: Journal for Research in Mathematics Education, Vol. 27 (1996), p. 521-539.

[4] E. Silver, J. Mamona-Downs and S. Leung: Journal for Research in Mathematics Education, Vol.27 (1996), p. 293-309.

[5] J. Smilansky: Journal of Educational Psychology, Vol.76 (1984), p. 377-386.

[6] R. Wicklein: The Technology Teacher, Vol.65 (2006), p. 25-29.

[7] C. L. Dym, A. M. Agogino, and O. Eris: Journal of Engineering Education, Vol.94 (2005), p. 103-120. 